\title{
On Riemannian Manifolds Admitting \\ a Certain Transformation
}

Dedicated to Professor Yoshie Katsurada on her 60th birthday

\author{
By Shigeyoshi Fujimura
}

\section{§ 0. Introduction.}

Let $\left(M^{n}, g\right)(n \geqq 2)$ be an $n$-dimensional Riemannian manifold ${ }^{1)}$ with a positive definite metric tensor $g$. The following is well known.

Theorem A (T. Nagano [7] $\left.{ }^{2)}\right)$. Let $\left(M^{n}, g\right)(n \geqq 4)$ be a Riemannian manifold. If $C$ does not vanish on $\left(M^{n}, g\right)$, there exists a metric tensor $g^{\prime}$ conformal to $g$ such that

$$
C\left(M^{n}, g\right)=I\left(M^{n}, g^{\prime}\right)
$$

where $C$ is the Weyl's conformal curvature tensor of $\left(M^{n}, g\right)$, and $C\left(M^{n}, g\right)$ and $I\left(M^{n}, g\right)$ are the groups of conformal transformations and isometric transformations of $\left(M^{n}, g\right)$, respectively.

From Theorem A, we can conjecture that $\left(M^{n}, g\right)$ is conformally flat if $C\left(M^{n}, g\right) \neq I\left(M^{n}, g\right)$ ([10]). In this respect, we can consider that if $\left(M^{n}, g\right)$ admits a transformation with a certain property, it shall restrict some geometrical properties of $\left(M^{n}, g\right)$. Taking the above into consideration, we shall study groups of homothetic transformations, conformal transformations and projective transformations.

The author wishes to express here his sincere thanks to Professor Yoshie Katsurada and Doctor Tamao Nagai for their kindly guidences and encouragements.

\section{§1. Homothetic transformations.}

Let $C\left(M^{n}, g\right), A\left(M^{n}, g\right), H\left(M^{n}, g\right)$ and $I\left(M^{n}, g\right)$ be groups of conformal transformations, affine transformations, homothetic transformations and isometric transformations of $\left(M^{n}, g\right)$, respectively. M. S. Knebelman and K. Yano ([4], [5], [13]) proved the followings.

Theorem B (K. Yano). In a compact Riemannian manifold $\left(M^{n}, g\right)$

1) Throughout the paper, we assume that $\left(M^{n}, g\right)$ is connected.

2) The numbers in brackets refer to references at the end of this paper. 
$(n \geqq 2)$, an infinitesimal affine transformation is an infinitesimal isometric transformation,

Theorem C (M. S. Knebelman and K. Yano). In a Riemannian manifold $\left(M^{n}, g\right)(n \geqq 2)$ of constant curvature (an Einstein manifold or a Riemannian manifold of constant scalar curvature), one of the following two conditions must be satisfied.

1) An infinitesimal homothetic transformation is an infinitesimal isometric transformation.

2) $R=0($ Ric $=0$ or $S=0)$,

where $R$, Ric and $S$ are the curvature tensor, the Ricci tensor and the scalar curvature of $\left(M^{n}, g\right)$, respectively.

The generalizations of Theorem B were studied by J. Hano [2], S. Ishihara and M. Obata [3] and S. Kobayashi [6], and concerning $H\left(M^{n}, g\right)$, the following theorem was proved.

Theorem D (S. Ishihara and M. Obata [3]). In a complete Riemannian manifold $\left(M^{n}, g\right)(n \geqq 2)$, one of the following two conditions must be satisfied.

1) $H\left(M^{n}, g\right)=I\left(M^{n}, g\right)$.

2) $\left(M^{n}, g\right)$ is locally flat.

In this section, we consider the case where $\left(M^{n}, g\right)$ satisfies some conditions on the curvature tensor instead of the completeness in Theorem D. We denote by $|T|_{g}$ the length of an arbitrary tensor $T$ with respect to $g$. The first theorem is the following.

THEOREM 1. If the curvature tensor $R$ of a Riemannian manifold $\left(M^{n}, g\right)(n \geqq 2)$ does not vanish on $\left(M^{n}, g\right)$, there exists a metric tensor $g^{\prime}$ conformal to $g$ such that $H\left(M^{n}, g\right)$ is implied in $I\left(M^{n}, g^{\prime}\right)$.

PROOF. In the same way as a proof of Theorem $\mathrm{A}$, we can prove this theorem. Let $g^{*}$ be a metric tensor on $M^{n}$ induced from $g$ by $f \in H\left(M^{n}, g\right)$ and $R^{*}$ the curvature tensor defined by $g^{*}$. Then, there exists a constant $c$ such that $g^{*}=e^{2 c} g$, and we have

$$
R^{*}=R, \quad\left|R^{*}\right|_{g^{*}}=e^{-2 c}|R|_{g}
$$

Therefore, defining $g^{\prime}$ by $|R|_{g} g$, we have

$$
f^{*}\left(g^{\prime}\right)=f^{*}\left(|R|_{g}\right) f^{*}(g)=g^{\prime},
$$

where $f^{*}$ denotes the dual mapping of the induced mapping $f_{*}$. The last equation shows that $f$ is an isometric transformation of $\left(M^{n}, g^{\prime}\right)$.

COROLLARY 1. If the Ricci tensor (the scalar curvature, the Weyl's conformal curvature tensor, or the Weyl's projective curvature tensor) does 
not vanish on a Riemannian manifold $\left(M^{n}, g\right)$, there exists a metric tensor $g^{\prime}$ conformal to $g$ such that $H\left(M^{n}, g\right)$ is implied in $I\left(M^{n}, g^{\prime}\right)$.

In connection with Theorem $\mathrm{C}$, we obtain the following.

THEOREM 2. If the length $|R|_{g}$ of the curvature tensor is bounded on a Riemannian manifold $\left(M^{n}, g\right)(n \geqq 2)$, one of the following two conditions must be satisfied.

1) $H\left(M^{n}, g\right)=I\left(M^{n}, g\right)$.

2) $\left(M^{n}, g\right)$ is locally flat.

Using the following lemma, we can prove this theorem.

Lemma 1. Let $\left(M^{n}, g\right)(n \geqq 2)$ be a Riemannian manifold which is not locally flat. If $H\left(M^{n}, g\right) \neq I\left(M^{n}, g\right)$, there exists a point $p_{0}$ of $\left(M^{n}, g\right)$ such that $R$ does not vanish at $p_{0}$, and for the point $p_{0}$ of $\left(M^{n}, g\right)$, the number of elements of the set

$$
M_{0}=\left\{f^{k}\left(p_{0}\right) \in M^{n} ; \quad k=0,1,2, \cdots\right\}
$$

is infinite, where $f^{k}=f \cdot f \cdots f(k$-times $)$ and $f^{0}=i d e n t i t y$.

Proof. The first half is obvious. For the latter half, we now assume that there exist two distinct numbers $i$ and $j$ such that

$$
f^{i}\left(p_{0}\right)=f^{j}\left(p_{0}\right) \text {, i.e., } f^{j-i}\left(p_{0}\right)=p_{0} .
$$

Therefore, from (1), we have

$$
|R|_{g}\left(p_{0}\right)=\left(\left(f^{j-i}\right)^{*}|R|_{g}\right)\left(p_{0}\right)=e^{-2 c(j-i)}|R|_{g}\left(p_{0}\right) .
$$

From $|R|_{g}\left(p_{0}\right) \neq 0$ and $i \neq j$, we have that $c$ is zero. This is contradictory to $H\left(M^{n}, g\right) \neq I\left(M^{n}, g\right)$.

Proof of Theorem 2. If $\left(M^{n}, g\right)$ is not locally flat and $H\left(M^{n}, g\right) \neq$ $I\left(M^{n}, g\right)$, there exists a point $p_{0}$ of $\left(M^{n}, g\right)$ and a negative constant $c$ such that $R$ does not vanish at $p_{0}$ and

$$
f^{*} g=e^{2 c} g \text { for a certain } f \in H\left(M^{n}, g\right) .
$$

On the other hand, we have

$$
\left(\left(f^{k}\right)^{*}|R|_{g}\right)\left(p_{0}\right)=e^{-2 c k}|R|_{g}\left(p_{0}\right) \quad(k=0,1,2, \cdots) .
$$

Therefore, from Lemma $1,|R|_{g}\left(p_{0}\right) \neq 0$ and $c<0$, we have

$$
\lim _{k \rightarrow+\infty}|R|_{g}\left(f^{k}\left(p_{0}\right)\right)=|R|_{g}\left(p_{0}\right) \lim _{k \rightarrow+\infty} e^{-2 c k}=+\infty .
$$

This is contradictory to the boundedness of $|R|_{g}$.

Corollary 2. In a locally symmetric Riemannian manifold $\left(M^{n}, g\right)$ 
$(n \geqq 2)$, one of the following two conditions must be satisfied.

1) $H\left(M^{n}, g\right)=I\left(M^{n}, g\right)$.

2) $\left(M^{n}, g\right)$ is locally flat.

In the same way as the proof of Theorem 2, we have the following theorem.

Theorem 3. If $\mid$ Ric $\left.\right|_{g}\left(S,|C|_{g}\right.$, or $\left.|P|_{g}\right)$ is bounded on a Riemannian manifold $\left(M^{n}, g\right)$, one of the following two conditions must be satisfied, where $P$ is the Weyl's projective curvature tensor.

1) $H\left(M^{n}, g\right)=I\left(M^{n}, g\right)$.

2) $R i c=0(S=0, C=0$, or $R=0)$.

\section{§2. Conformal transformations.}

In the same way as the proof of Theorem 2, we obtain the following.

THEOREM 4. Let $\left(M^{n}, g\right)(n \geqq 4)$ be a Riemannian manifold such that the length of the Weyls' conformal curvature tensor is bounded on it. If there exists a conformal transformation $f$ such that

$$
f^{*} g=e^{2 \rho} g \text { and }|\rho|>\varepsilon>0
$$

for a certain positive constant $\varepsilon$, then, $\left(M^{n}, g\right)$ is conformally flat.

Corollary 3 . In a compact Riemannian manifold $\left(M^{n}, g\right)(n \geqq 4)$, if there exists a conformal transformation $f$ such that

$$
f^{*} g=e^{2 \rho} g \text { and } \rho \neq 0 \text { on }\left(M^{n}, g\right),
$$

then, $\left(M^{n}, g\right)$ is conformally flat.

Let $\left(M^{n}, g\right)$ and $\left(\bar{M}^{n}, \bar{l}\right)(n \geqq 2)$ be two Riemannian manifolds. A correspondence $f$ from $\left(M^{n}, g\right)$ to $\left(\bar{M}^{n}, \bar{g}\right)$ is conformal if and only if there exists a function $\rho$ defined on $\left(M^{n}, g\right)$ such that

$$
f^{*} \bar{g}=e^{2 o} g .
$$

Then, we have the following lemma.

Lemma 2. Let $\left(M^{n}, g\right)$ and $\left(\bar{M}^{n}, \bar{g}\right)(n \geqq 4)$ be two Riemannian manifolds. If there exists a conformal correspondence $f$ from $\left(M^{n}, g\right)$ to $\left(\bar{M}^{n}, \bar{g}\right)$, i.e., $f^{*} \bar{g}=e^{2 p} g$, one of the following two conditions must be satisfied at each point of $\left(M^{n}, g\right)$.

1) $C=0$, if $\bar{C}=0$.

2) $\boldsymbol{\rho}=\frac{1}{2}\left(\log |C|_{g}-\log \left|\bar{C}^{*}\right|_{\bar{g}^{*}}\right)$, if $\bar{C} \neq 0$,

where $\bar{g}^{*}$ and $\bar{C}^{*}$ are a metric tensor on $M^{n}$ induced by $f$ from $\bar{g}$ and the Weyl's conformal curvature tensor defined by $y^{*}$. 
ProOF. From $\bar{g}^{*}=e^{2 \rho} g$, we have

$$
\bar{C}^{*}=C \quad \text { and } \quad\left|\bar{C}^{*}\right|_{\bar{g}^{*}}=e^{-2 \rho}|C|_{g} .
$$

From (2) and $f^{*} \bar{C}=\bar{C}^{*}$, this lemma is obvious.

From Lemma 2, we have the following.

Corollary 4. Let $\left(M^{n}, g\right)$ and $\left(\bar{M}^{n}, \bar{g}\right)(n \geqq 4)$ be two Riemannian manifolds such that the lengths of the Weyl's conformal curvature tensors of $\left(M^{n}, g\right)$ and $\left(\bar{M}^{n}, \bar{g}\right)$ are constant. If there exists a conformal correspondence $f$ from $\left(M^{n}, g\right)$ to $\left(\bar{M}^{n}, \bar{g}\right)$, one of the following two conditions must be satisfied.

1) $\left(M^{n}, g\right)$ and $\left(\bar{M}^{n}, \bar{g}\right)$ are conformally flat.

2) $f$ is homothetic.

This corollary implies the result obtained by T. Adati and T. Miyazawa [1].

Proof. Since $|\overline{\boldsymbol{C}}|_{\bar{g}}$ is constant,

$$
\left|\bar{C}^{*}\right|_{\bar{g}^{*}}=f^{*}\left(|\bar{C}|_{\bar{g}}\right)=|\bar{C}|_{\bar{g}}=\text { constant. }
$$

Therefore, from Lemma 2 , if $C$ is zero at a certain point of $\left(M^{n}, g\right),\left(M^{n}, g\right)$ and $\left(\bar{M}^{n}, \bar{g}\right)$ are conformally flat, otherwise, $\rho$ is constant.

Corollary 5 (T. Sumitomo [12]). Let $\left(M^{n}, g\right)(n \geqq 4)$ be a Riemannian manifold such that the length of the Weyl's conformal curvatature tensor is constant. Then, one of the following two conditions must be satisfied.

1) $\left(M^{n}, g\right)$ is conformally flat.

2) $C\left(M^{n}, g\right)=I\left(M^{n}, g\right)$.

PROOF. Denoting by $g^{*}$ a metric tensor on $M^{n}$ induced by $f$ from $g$ for $f \in C\left(M^{n}, g\right)$, since $|C|_{g}$ is constant, we have

$$
\left|C^{*}\right|_{g^{*}}=f^{*}\left(|\mathrm{C}|_{g}\right)=|C|_{g} .
$$

Therefore, if $C \neq 0$, we have that $\rho$ is zero.

Next, we shall consider the relation between the curvatures and the conformal correspondences of two Riemannian manifolds. The following theorem is well known.

TheOREM E (M. Obata [9]). There is no conformal correspondence between a compact Riemannian manifold of everywhere nonpositive scalar curvature and one of everywhere nonnegative scalar curvature except for the case where both scalar curvatures are identically zero.

With respect to the relations between the sectional curvatures and the conformal correspondence of two Riemannian manifolds, we have the following theorem which is similar to the above Theorem E. 
Theorem 5. Let $\left(M^{n}, g\right)(n \geqq 2)$ be a compact Riemannian manifold of everywhere nonpositive sectional curvature and $\left(\bar{M}^{n}, \bar{g}\right)$ one of everywhere nonnegative sectional curvature. If there exists a conformal correspondence $f$ from $\left(M^{n}, g\right)$ to $\left(\bar{M}^{n}, \bar{g}\right)$, then, $\left(M^{n}, g\right)$ and $\left(\bar{M}^{n}, \bar{g}\right)$ are locally flat and $f$ is homothetic.

Proof. Let $\bar{g}^{*}, \vec{R}^{*}$ and $\bar{K}^{*}$ be the metric tensor, the curvature tensor and the sectional curvature induced by $f$ from $\bar{g}, \bar{R}$ and $\bar{K}$, respectively, where $K$ is a sectional curvature of $\left(M^{n}, g\right)$ and $\bar{K}$ one of $\left(\bar{M}^{n}, \bar{g}\right)$. Denoting by $\left\{u_{1}, \cdots, u_{n}\right\}$ the orthonormal basis of the tangent space of $\left(M^{n}, g\right)$ at each point with respect to $g$ and by $P_{i j}$ the plane expanded by $u_{i}$ and $u_{j}$, we have

$$
K\left(P_{i j}\right)=\frac{g\left(R\left(u_{i}, u_{j}\right) u_{i}, u_{j}\right)}{g\left(u_{i}, u_{i}\right) g\left(u_{j}, u_{j}\right)-\left(g\left(u_{i}, u_{j}\right)\right)^{2}}=g\left(R\left(u_{i}, u_{j}\right) u_{i}, u_{j}\right)
$$

and

$$
\bar{K}^{*}\left(P_{i j}\right)=\frac{\bar{g}^{*}\left(\bar{R}^{*}\left(u_{i}, u_{j}\right) u_{i}, u_{j}\right)}{\bar{g}^{*}\left(u_{i}, u_{i}\right) \bar{g}^{*}\left(u_{j}, u_{j}\right)-\left(\bar{g}^{*}\left(u_{i}, u_{j}\right)\right)^{2}}=e^{-2 \rho} g\left(\ddot{R}^{*}\left(u_{i}, u_{j}\right) u_{i}, u_{j}\right),
$$

where $\rho$ is an associated function with $f$. On the other hand, using local coordinates, we have

$$
\bar{R}^{*{ }_{i j k}}=R_{i j k}^{h}-\delta_{k}^{h} \rho_{i j}+\delta_{j}^{h} \rho_{i k}-g_{i j} \rho^{h}{ }_{k}+g_{i k} \rho^{h}{ }_{j},
$$

where $\rho_{i}=\partial_{i} \rho, \rho_{i}^{h}=g^{h j} \rho_{j i}$ and

$$
\boldsymbol{\rho}_{i j}=\boldsymbol{\nabla}_{j} \boldsymbol{\rho}_{i}-\boldsymbol{\rho}_{i} \boldsymbol{\rho}_{j}+\frac{1}{2} g^{k l} \boldsymbol{\rho}_{k} \boldsymbol{\rho}_{i} g_{i j},
$$

and $\nabla_{i}$ is a covariant differential operator defined by $g$ with respect to $\frac{\partial}{\partial x^{i}}$. Therefore, from (3), (4) and (5), we have

$$
e^{2 \rho} \bar{K}^{*}\left(P_{i j}\right)=K\left(P_{i j}\right)+\left(u_{i}^{k} u_{i}^{l}+u_{j}^{k} u_{j}^{l}\right) \rho_{k l},
$$

where $u_{i}=u_{i}^{k} \frac{\partial}{\partial x^{k}}$. From (6), we obtain the following system of partial differential equations.

$$
\left(u_{i}^{k} u_{i}^{l}+u_{j}^{k} u_{j}^{l}\right) \frac{\partial^{2} \rho}{\partial x^{k} \partial x^{l}}+\left(g^{k l} \rho_{k}+A_{i j}^{l}\right) \rho_{l}=e^{2 \rho} \bar{K}^{*}\left(P_{i j}\right)-K\left(P_{i j}\right),
$$

where $A_{i j}^{l}$ is denoted by

$$
-\left(u_{i}^{h} u_{i}^{k}+u_{j}^{h} u_{j}^{k}\right)\left\{\begin{array}{l}
l \\
h k
\end{array}\right\}-\left(u_{i}^{h} u_{i}^{l}+u_{j}^{h} u_{j}^{l}\right) \rho_{h}
$$

and $\left\{\begin{array}{l}l \\ h k\end{array}\right\}$ are the coefficients of the connection defined by $g$. Therefore, for 
$n$ planes $P_{12}, P_{23}, \cdots, P_{n-1, n}$ and $P_{n 1}$, from (7), we have

$$
\begin{aligned}
& 2\left(\sum_{i=1}^{n} u_{i}^{k} u_{i}^{l}\right) \frac{\partial^{2} \rho}{\partial x^{k} \partial x^{l}}+\left(\sum_{i=1}^{n-1} A_{i, i+1}^{l}+A_{n 1}^{l}\right) \rho_{l} \\
& \quad=e^{2 \rho}\left\{\sum_{i=1}^{n-1} \bar{K}^{*}\left(P_{i, i+1}\right)+\bar{K}^{*}\left(P_{n 1}\right)\right\}-\left\{\sum_{i=1}^{n-1} K\left(P_{i, i+1}\right)+K\left(P_{n 1}\right)\right\} .
\end{aligned}
$$

On the other hand, since $\left(\sum_{i=1}^{n} u_{i}^{k} u_{i}^{l}\right)$ is positive definite, applying Theorem of E. Hopf [14] to (8), we have that $\rho$ is constant, and $\bar{K}^{*}$ and $K$ are identically zero.

Corollary 6. Let $\left(M^{n}, g\right)$ and $\left(\bar{M}^{n}, \bar{g}\right)(n \geqq 2)$ be two compact Riemannian manifolds of constant curvature. If there exists a conformal correspondence $f$ between $\left(M^{n}, g\right)$ and $\left(\bar{M}^{n}, \bar{g}\right)$, one of the following two conditions must be satisfied.

1) The sectional curvatures of $\left(M^{n}, g\right)$ and $\left(\bar{M}^{n}, \bar{g}\right)$ are of the same sign.

2) $f$ is homothetic and $\left(M^{n}, g\right)$ and $\left(\bar{M}^{n}, \bar{g}\right)$ are locally flat.

\section{$\S 3 . \quad$ Projective transformations.}

K. Yano and T. Nagano [15] and T. Sumitomo [12] proved the following theorems.

Theorem F (K. Yano and T. Nagano). Let $\left(M^{n}, g\right)(n \geqq 3)$ be a locally symmetric Riemannian manifold. If $\left(M^{n}, g\right)$ admits an infinitesimal projective transformation $X$, one of the following two conditions must be satisfied.

1) $\left(M^{n}, g\right)$ is of constant curvature.

2) $X$ is an infinitesimal affine transformation.

Theorem G (T. Sumitomo). Let $\left(M^{n}, g\right)(n \geqq 2)$ be a Ricci symmetric Riemannian manifold. If $\left(M^{n}, g\right)$ admits an infinitesimal projective transformation $X$, one of the following two conditions must be satisfied.

1) $\left(M^{n}, g\right)$ is an Einstein manifold.

2) $X$ is an infinitesimal affine transformation.

In connection with Theorem F, N. S. Sinyukov [11] gave the analogous result in the case where there exists a projective correspondence between a locally symmetric Riemannian manifold and an arbitrary Riemannian manifold, and T. Nagano [8] obtained a similar result in the case where there exists a projective correspondence between two complete Ricci symmetric Riemannian manifolds. In this section, we shall prove the following theorems.

THEOREM 6. Let $\left(M^{n}, g\right)(n \geqq 4)$ be a conformally flat Riemannian manifold of constant scalar curvature and $\left(\bar{M}^{n}, \bar{g}\right)$ a Riemannian manifold such that the tensor $\overline{\bar{V}} \vec{R} i c$ is symmetric, where $\bar{\nabla}$ is a covariant differential operator 
with respect to $\bar{g}$. If there exists a projective correspondence $f$ from $\left(M^{n}, g\right)$ to $\left(\bar{M}^{n}, \bar{g}\right)$, one of the following two conditions must be satisfied.

1 ) $\left(M^{n}, g\right)$ and $\left(\bar{M}^{n}, \bar{g}\right)$ are of constant curvature.

2) $f$ is an affine correspondence.

Theorem 7. Let $\left(M^{n}, g\right)$ and $\left(\bar{M}^{n}, \bar{g}\right)(n \geqq 2)$ be two Ricci symmetric Riemannian manifolds. If there exists a projective correspondence $f$ from $\left(M^{n}, g\right)$ to $\left(\bar{M}^{n}, \bar{g}\right)$, one of the following two conditions must be satisfied.

1) $\left(M^{n}, g\right)$ and $\left(\bar{M}^{n}, \bar{g}\right)$ are Einstein manifolds.

2) $f$ is an affine correspondence.

Lemma 3. In a conformally flat Riemannian manifold $\left(M^{n}, g\right)(n \geqq 3)$, if there exists a vector field $\phi_{i}$ on $\left(M^{n}, g\right)$ such that

$$
\phi_{h} P_{i j k}^{h}=0,
$$

then, $\left(M^{n}, g\right)$ is of constant curvature, or $\phi_{i}$ is a zero vector field, where $P_{i j k}^{h}$ are components of $P$ with respect to local coordinates.

Proof. From the definition of $P$ and (9), we have

$$
\phi_{h} R_{i j k}^{n}=\frac{1}{n-1}\left(\phi_{k} R_{i j}-\phi_{j} R_{i k}\right)
$$

Contracting (10) by $g^{i j}$, we have

$$
\phi_{h} R_{k}^{h}=\frac{1}{n} S \phi_{k} .
$$

On the other hand, since $\left(M^{n}, g\right)$ is conformally flat,

$$
\begin{aligned}
R^{h}{ }_{i j k}= & \frac{1}{n-2}\left(\delta_{k}^{h} R_{i j}-\delta_{j}^{h} R_{i k}+g_{i j} R_{k}^{h}-g_{i k} R^{h}{ }_{j}\right) \\
& -\frac{1}{(n-1)(n-2)} S\left(\delta_{k}^{h} g_{i j}-\delta_{j}^{h} g_{i k}\right)
\end{aligned}
$$

Substituting (12) into (10) and using (11), we have

$$
\phi_{k} R_{i j}-\psi_{j} R_{i k}-\frac{1}{n} S\left(\phi_{k} g_{i j}-\psi_{j} g_{i k}\right)=0 .
$$

Contracting (13) by $g^{k l} \psi_{l}$ and using (11), we have

$$
\left(g^{k l} \psi_{k} \psi_{l}\right)\left(R_{i j}-\frac{1}{n} S g_{i j}\right)=0 .
$$

Therefore, $\left(M^{n}, g\right)$ is conformally flat and Einsteinian, i. e., of constant curvature, or $\phi_{i}$ is a zero vector field. 
PRoOF of Theorem 6. Since $\left(M^{n}, g\right)$ is conformally flat, from (12) and the Bianchi's identity, we have

$$
\nabla_{k} R_{i j}-\nabla_{j} R_{i k}-\frac{1}{2(n-1)}\left(g_{i j} \nabla_{k} S-g_{i k} \nabla_{j} S\right)=0 .
$$

Since $S$ is constant, from (14), we have

$$
\nabla_{k} R_{i j}-\nabla_{j} R_{i k}=0 .
$$

When we denote by $\tilde{\nabla}, \widetilde{R}, \widetilde{R} i c$ and $\widetilde{P}$ the covariant differential operator, the curvature tensor, the Ricci tensor and the Weyl's projective curvature tensor induced by $f$ from $\bar{\nabla}, \bar{R}, \bar{R} i c$ and $\bar{P}$, respectively, it follows that

$$
\tilde{\nabla}_{k} \widetilde{R}_{i j}-\widetilde{\nabla}_{j} \widetilde{R}_{i k}=0,
$$

because $\bar{\nabla} \bar{R} i c$ is a symmetric tensor. On the other hand,

$$
\begin{aligned}
\tilde{\nabla}_{l} \widetilde{p}^{h}{ }_{i j k}= & \nabla_{l} P^{n}{ }_{i j k}+\delta_{l}^{h} \psi_{m} P^{m}{ }_{i j k}-2 \psi_{l} P^{n}{ }_{i j k} \\
& -\psi_{i} P^{n}{ }_{l j k}-\psi_{j} P^{n}{ }_{i l k}-\psi_{k} P^{n}{ }_{i j l},
\end{aligned}
$$

where $\phi_{i}$ is a covariant vector field associated with $f$. Contracting (17) by $\delta_{h}^{l}$, we have

$$
\tilde{\nabla}_{h} \widetilde{p}_{i j k}^{h}=\nabla_{h} P^{h}{ }_{i j k}+(n-2) \psi_{h} P_{i j k}^{h} .
$$

From (15) and (16), we have

$$
\tilde{\nabla}_{h} \widetilde{p}_{i j k}^{h}=\nabla_{h} P_{i j k}^{h}=0
$$

Substituting (19) into (18), for $n \geqq 3$, we have (9). Therefore, from Lemma 3 , we have that $\left(M^{n}, g\right)$ is of constant curvature, or $\phi_{i}$ is a zero vector field (i. e., $f$ is an affine correspondence). When $\left(M^{n}, g\right)$ is of constant curvature, $\left(\bar{M}^{n}, \bar{g}\right)$ is also of constant curvature.

Corollary 7 . Let $\left(M^{n}, g\right)(n \geqq 4)$ be a conformally flat Riemannian manifold of constant scalar curvature. If $\left(M^{n}, g\right)$ admits a projective transformation $f$, then, $\left(M^{n}, g\right)$ is of constant curvature, or $f$ is an affine transformation.

Lemma 4. Let $\left(M^{n}, g\right)(n \geqq 2)$ be a Riemannian manifold satisfying

$$
\nabla_{l} \nabla_{k} R_{i j}-\nabla_{k} \nabla_{l} R_{i j}=0 .
$$

If there exists a vector field $\phi_{i}$ satisfying (9), then, $\left(M^{n}, g\right)$ is an Einstein manifold, or $\psi_{i}$ a zero vector field.

Proof. From (9), we have (10) and (11). Contracting (10) by $g^{k l} \phi_{l} R^{i j}$, we have 


$$
g^{k l} \psi_{l} \psi_{h} R^{i j} R_{i j k}^{h}=\frac{1}{n-1}\left(g^{k l} \phi_{k} \psi_{l} R^{i j} R_{i j}-\psi_{j} \psi_{k} R_{i}^{j} R^{i k}\right)
$$

On the other hand, from (20) and the Ricci's identity,

$$
R_{h j} R_{i k l}^{h}+R_{i l} R^{h}{ }_{j k l}=0 .
$$

Contracting (22) by $g^{i l}$ and substituting the result and (11) into (21), we have

$$
g^{k l} \phi_{k} \psi_{l}\left(R_{i j} R^{i j}-\frac{1}{n} S^{2}\right)=0 .
$$

Therefore, it follows from (23) that

$$
R_{i j}-\frac{1}{n} S g_{i j}=0, \text { or } \quad \phi_{i}=0 \text {. }
$$

Proof of Theorem 7. In the same way as the proof of Theorem 6, since $\nabla$ Ric and $\bar{\nabla} \vec{R} i c$ are zero tensors, we have (9) and (20). Therefore, from Lemma 4 , it follows that $\left(M^{n}, g\right)$ is an Einstein manifold, or $f$ is an affine correspondence. Similarly, we have that $\left(\bar{M}^{n}, \bar{g}\right)$ is an Einstein manifold when $f$ is not an affine correspondence.

Addendum. Mr. T. Muramori indicated the author that we have the following theorem in the same way as Theorem 2 .

Theorem 8. If $|\nabla R|$ is bounded on $\left(M^{n}, g\right)$, one of the following two conditions must be satisfied.

1) $H\left(M^{n}, g\right)=I\left(M^{n}, g\right)$.

2) $\left(M^{n}, g\right)$ is locally symmetric.

We can generalize this theorem as follows:

Lemma 5. Suppose that $H\left(M^{n}, g\right)$ is not equal to $I\left(M^{n}, g\right)$. If a tensor $T$ satisfies that there exists a number $m$ such that $\left|\nabla^{m} T\right|$ is bounded on $\left(M^{n}, g\right)$ and

$$
f^{*}\left(\left|\nabla^{k} T\right|\right)=e^{2(k+1) c}\left|\nabla^{k} T\right| \quad \text { for all } k(k=0, \cdots, m)
$$

then, $T$ is a zero tensor, where $\nabla^{m}$ is a covariant derivative of $m$ times, $c$ is a non-zero constant and $f$ is in $H\left(M^{n}, g\right)$ such that $f^{*} g=e^{2 c} g$.

This lemma can be proved by using a method of the proof of Theorem 2 and that $\left|\nabla^{m-1} T\right|$ is constant. From this lemma, we have the followings.

THEOREM 9. If there exists a number $m$ such that $\left|\nabla^{m} R\right|\left(\left|\nabla^{m} P\right|\right.$, or $\left.\left|\nabla^{m} Z\right|\right)$ is bounded on $\left(M^{n}, g\right)$, one of the following two conditions must be satisfied,

1) $H\left(M^{n}, g\right)=I\left(M^{n}, g\right)$.

2) $\left(M^{n}, g\right)$ is locally symmetric, 
where $Z_{i j k}^{h}=R_{i j k}^{h}-S\left(\delta_{k}^{h} g_{i j}-\delta_{j}^{h} g_{i k}\right) / n(n-1)$.

THEOREM 10. If there exists a number $m$ such that $\left|\nabla^{m} C\right|$ is bounded on $\left(M^{n}, g\right)$, one of the following two conditions must be satisfied.

1) $H\left(M^{n}, g\right)=I\left(M^{n}, g\right)$.

2) $\left(M^{n}, g\right)$ is conformally flat.

THEOREM 11. If there exists a number $m$ such that $\left|\nabla^{m} \operatorname{Ric}\right|$, or $\left|\nabla^{n} G\right|$, is bounded on $\left(M^{n}, g\right)$, one of the following two conditions must be satisfied.

1) $H\left(M^{n}, g\right)=I\left(M^{n}, g\right)$.

2) $\left(M^{n}, g\right)$ is an Einstein manifold of zero scalar curvature, where $G_{i j}=R_{i j}-S \cdot g_{i j} / n$.

THEOREM 12. If there exists a number $m$ such that $\left|\nabla^{m} S\right|$ is bounded on $\left(M^{n}, g\right)$, one of the following two conditions must be satisfied.

1) $H\left(M^{n}, g\right)=I\left(M^{n}, g\right)$.

2) $\left(M^{n}, g\right)$ is of zero scalar curvature.

Department of Mathematics,

Hokkaido University

\section{References}

[1] T. AdAti and T. Miyazawa: On conformally symmetric spaces, Tensor (N.S.), 18 (1967), 335-342.

[2] J. HANO: On affine transformations of a Riemannian manifold, Nagoya Math. J., 9 (1955), 99-106.

[3] S. IshiHARA and M. OBATA: Affine transformations in a Riemannian manifold, Tôhoku Math. J., 7 (1955), 146-150.

[4] M. S. Knebelman: Homothetic mappings of Riemann spaces, Proc. Amer. Math. Soc., 9 (1958), 926-927.

[5] M. S. KNEBElman and K. Yano: On homothetic mappings of Riemann spaces, Proc. Amer. Math. Soc., 12 (1961), 300-303.

[6] S. KobAyASHI: A theorem on the affine transformation group of a Riemannian manifold, Nagoya Math. J., 9 (1955), 39-41.

[7] T. NAGANO: On conformal transformations of Riemannian spaces, J. Math. Soc. Japan, 10 (1958), 79-93.

[8] T. NAGANO: The projective transformation on a space with Parallel Ricci tensor, Kōdai Math. Sem. Rep., 11 (1959), 131-138.

[9] M. OBATA: Conformal transformations of compact Riemannian manifolds, Illinois J. Math., 6 (1962), 292-295.

[10] M. OBATA: Conformal transformations of Riemannian manifolds, Sugaku, 14 (1963), 152-164, (in Japanese).

[11] N. S. Sinyukov: On the geodesic correspondence of a Riemannian space with a symmetric space, Dokl. Akad. Nauk, SSSR, 98 (1954) 21-23. 
[12] T. Sumitomo: Projective and conformal transformations in compact Riemannian manifolds, Tensor (N. S.), 9 (1959), 113-135.

[13] K. Yano: On harmonic and Killing vector fields, Ann. Math, 55 (1952), 38-45.

[14] K. Yano and S. Bochner: Curvatures and Betti numbers, Ann. Math. Studies, No. 32, Princeton, 1953.

[15] K. YANO and T. NAGANO: Some theorems on projective and conformal transformations, Indag. Math., 19 (1957), 451-458.

(Received March 26, 1971) 